\title{
GLOBAL BEHAVIOR OF INTEGRAL TRANSFORMS
}

\author{
JASSON VINDAS AND RICARDO ESTRADA
}

Received 23 August 2005; Revised 13 December 2005; Accepted 29 December 2005

We obtain global estimates for various integral transforms of positive differentiable functions that satisfy inequalities of the type $c_{1} f(x) / x \leq-f^{\prime}(x) \leq c_{2} f(x) / x$, for $x>0$.

Copyright (c) 2006 J. Vindas and R. Estrada. This is an open access article distributed under the Creative Commons Attribution License, which permits unrestricted use, distribution, and reproduction in any medium, provided the original work is properly cited.

\section{Introduction}

In a recent article, Berndt [1] obtained the following global estimate for the Fourier sine transform of the function $f$ :

$$
\frac{A}{x} f\left(\frac{1}{x}\right) \leq \int_{0}^{\infty} f(u) \sin (u x) d u \leq \frac{B}{x} f\left(\frac{1}{x}\right), \quad \forall x>0,
$$

where $A$ and $B$ are positive constants, provided that $f$ is a differentiable function defined on $(0, \infty)$ that satisfies

$$
c_{1} \frac{f(x)}{x} \leq-f^{\prime}(x) \leq c_{2} \frac{f(x)}{x}
$$

where $c_{1}$ and $c_{2}$ are constants with

$$
0<c_{1} \leq c_{2}<2
$$

It should be remarked that asymptotic estimates of the behavior of the sine and of other integral transforms of regularly varying functions [6] in terms of the function $f(1 / x)$ had been obtained before [7-9], both as $x \rightarrow 0$ and as $x \rightarrow \infty$. However, (1.1) is a global estimate that not only considers the endpoint behavior but also holds for all $x>0$.

Our aim in this article is to generalize (1.1) in two directions. On the one hand, we want to consider other kernels than sine, so we will give conditions on the kernel $k(x)$ 
such that an estimate of the form

$$
\frac{A^{\prime}}{x} f\left(\frac{1}{x}\right) \leq \int_{0}^{\infty} f(u) k(u x) d u \leq \frac{B^{\prime}}{x} f\left(\frac{1}{x}\right), \quad \forall x>0,
$$

holds if $f$ satisfies (1.2).

On the other hand, we will remove the condition $c_{2}<2$ for the sine transform. Actually, this condition was imposed by Berndt to guarantee the integrability of $\sin (u x) f(x)$ at $x=0$; if $c_{2} \geq 2$, this function might not be integrable near $x=0$. In such a case, the ordinary sine transform of $f$ will not exist, but one may consider regularizations of $f$ which are tempered distribution of the space $\mathscr{S}^{\prime}$, and whose Fourier sine transforms satisfy a global estimate as in (1.1), modulo a polynomial. In this way, we remove the problem of nonintegrability at $x=0$. We are also able to remove the integrability condition (in general, if $c_{2} \geq 1, f$ may not be integrable at 0 ) and obtain global estimates modulo a polynomial for the Laplace transform of $f$.

Our analysis is based on a characterization of the class of function $\mathfrak{V}$, which consists of those differentiable functions that satisfy (1.2). This characterization is given in Section 3. Using this characterization we are able to give several global estimates for integral transforms of elements of $\mathfrak{V}$, both for general oscillatory kernels, particularly for the sine transform, and for the Laplace transform in Sections 4 and 5.

\section{Preliminaries}

In this section, we explain the spaces of test functions and distributions employed in this article. We also present some of the properties of these spaces that will be needed in our analysis.

The space $\mathscr{S}$ of test functions of rapid decay and its dual space $\mathscr{S}^{\prime}$, space of tempered distributions, are well known $[4,5,10,11]$.

We will discuss the concept of regularization $[4,5,11]$. If $f$ is a function, denote by supp $f$ the closure of the set of points for which $f$ does not vanish. Let $f$ be a real-valued function, which we assume to be locally integrable in $\mathbb{R} \backslash\{0\}$; we say that a distribution $\tilde{f} \in \mathscr{S}^{\prime}$ is a regularization of $f$ at 0 if for all $\phi \in \mathscr{S}$ with supp $\phi \subseteq(-\infty, 0) \cup(0, \infty)$, we have

$$
\langle\tilde{f}(x), \phi(x)\rangle=\int_{-\infty}^{\infty} f(x) \phi(x) d x .
$$

Of course, we assume that the integral in the right of the last equality makes sense. The function $f$ has a regularization at $x=0$ if and only if it has an algebraic growth near the origin in the Cesàro sense [3] (see also [4, pages 297-332] for a complete discussion of Cesàro behavior of distributions). If a function $f$ has a regularization at 0 , then it has infinitely many regularizations at 0 , and all of them are obtained by adding a linear combination of the Dirac delta function and its derivatives concentrated at $0[4,10,11]$. Thus, given $\tilde{f}$ and $\tilde{f}_{1}$, two regularizations of $f$ at 0 , they satisfy

$$
\tilde{f}_{1}(x)=\tilde{f}(x)+\sum_{i=0}^{n} a_{i} \delta^{(i)}(x),
$$

for some real constants $a_{0}, \ldots, a_{n}$. 
Let $T$ be a linear continuous operator on $\mathscr{Y}$. We define its transpose as the linear operator ${ }^{\perp} T$ defined on $\mathscr{S}^{\prime}$ given by $g \mapsto^{\perp} T g$, where ${ }^{\perp} T g$ is the tempered distribution defined by

$$
\left\langle\left({ }^{\perp} T g\right)(x), \phi(x)\right\rangle=\langle g(x),(T \phi)(x)\rangle .
$$

The Fourier transform of a tempered distribution is defined as the transpose operator of the Fourier transform on the space of test functions of rapid decay at $\infty$ [10]. If $\phi \in \mathscr{Y}$, then its Fourier transform is again an element of $\mathscr{S}[4,5,10,11]$. Therefore, if $g \in \mathscr{Y}^{\prime}$, we define its Fourier transform $G \in \mathscr{Y}^{\prime}$ as

$$
\langle G(x), \phi(x)\rangle=\left\langle g(x), \int_{-\infty}^{\infty} \phi(u) e^{i x u} d u\right\rangle .
$$

We will define the sine transform of a tempered distribution in the same way as we defined the Fourier transform. Note that if $\phi \in \mathscr{Y}$, then its sine transform, defined as

$$
\int_{0}^{\infty} \phi(u) \sin (x u) d u
$$

is also an element of $\mathscr{Y}$. We define the sine transform on $\mathscr{S}^{\prime}$ as the transpose of the sine transform on $\mathscr{S}$.

The Laplace transform of a tempered distribution cannot be defined in every case. However, it can be defined for tempered distributions whose support is bounded on the left [11, pages 222-224]. In fact, if $g \in \mathscr{S}^{\prime}$ with supp $g \subseteq[0, \infty)$, we define $L$, the Laplace transform of $g$, as the function

$$
L(x)=\left\langle g(u), \lambda(u) e^{-x u}\right\rangle,
$$

where $\lambda$ is any infinitely smooth function with support bounded on the left, which equals one over a neighborhood of the support of $g$ [11]. This definition is independent of the choice of $\lambda$.

\section{Characterization of the class $\mathfrak{V}$}

In this section, we will define and characterize the class of functions $\mathfrak{V}$. The study of integral transforms of elements in this class will be the central subject of this paper.

Definition 3.1. A positive, differentiable function $f$ defined on $(0, \infty)$ is said to be an element of $\mathfrak{V}$ if it satisfies

$$
c_{1} \frac{f(x)}{x} \leq-f^{\prime}(x) \leq c_{2} \frac{f(x)}{x}
$$

where $c_{1}$ and $c_{2}$ are positive numbers.

We will prove that the functions in $\mathfrak{V}$ satisfy a variational property. Let us start by setting

$$
\epsilon(t)=\frac{-t f^{\prime}(t)}{f(t)}
$$


4 Global behavior of integral transforms

It follows that $\epsilon$ satisfies

$$
c_{1} \leq \epsilon(t) \leq c_{2}, \quad \forall t>0 .
$$

By integrating $-\epsilon(t) / t$, we obtain

$$
\log f(x)=-\int_{1}^{x} \frac{\epsilon(t)}{t} d t+\log f(1)
$$

and hence

$$
f(x)=f(1) \exp \left\{-\int_{1}^{x} \frac{\epsilon(t)}{t} d t\right\}
$$

which gives us a representation formula for $f$. Conversely, if (3.3) and (3.5) hold, then $f$ satisfies (3.1). This fact is stated in the following lemma.

Lemma 3.2. A function $f$ defined on $(0, \infty)$ belongs to the class $\mathfrak{V}$ if and only if it satisfies (3.5), where $\epsilon$ satisfies (3.3).

In fact, the last lemma was obtained by Berndt independently in his dissertation [2, Lemma 1.4]. We now give another characterization of the elements of $\mathfrak{V}$.

Theorem 3.3. A function $f$ defined on $(0, \infty)$ belongs to $\mathfrak{V}$ if and only if it is a positive differentiable function and satisfies

$$
\begin{gathered}
\frac{1}{u^{c_{1}}} \leq \frac{f(u x)}{f(x)} \leq \frac{1}{u^{c_{2}}}, \quad \forall x \in(0, \infty), \forall u \in(0,1], \\
\frac{1}{u^{c_{2}}} \leq \frac{f(u x)}{f(x)} \leq \frac{1}{u^{c_{1}}}, \quad \forall x \in(0, \infty), \forall u \in[1, \infty) .
\end{gathered}
$$

Proof. We assume that $f \in \mathfrak{V}$. By Lemma 3.2,

$$
f(x)=f(1) \exp \left\{-\int_{1}^{x} \frac{\epsilon(t)}{t} d t\right\}
$$

where $c_{1} \leq \epsilon(t) \leq c_{2}$. Therefore,

$$
\frac{f(u x)}{f(x)}=\exp \left\{\int_{1}^{x} \frac{\epsilon(t)}{t} d t-\int_{1}^{x u} \frac{\epsilon(t)}{t} d t\right\} .
$$

Let us take $u \in(0,1]$. Then we have

$$
\int_{1}^{x} \frac{\epsilon(t)}{t} d t-\int_{1}^{x u} \frac{\epsilon(t)}{t} d t=\int_{x u}^{x} \frac{\epsilon(t)}{t} d t
$$

Moreover,

$$
\log \left(\frac{1}{u^{c_{1}}}\right)=c_{1} \int_{x u}^{x} \frac{d t}{t} \leq \int_{x u}^{x} \frac{\epsilon(t)}{t} d t \leq c_{2} \int_{x u}^{x} \frac{d t}{t}=\log \left(\frac{1}{u^{c_{2}}}\right) .
$$

Therefore, (3.6) holds. By using a similar argument, we can see that (3.7) follows. 
Let us now assume the converse. First of all, we will show that $f$ is a decreasing function. Let us take $y \geq x$; by setting $u=x / y$ in (3.6), we obtain

$$
\frac{f(x)}{f(y)}=\frac{f(y(x / y))}{f(y)} \geq\left(\frac{x}{y}\right)^{-c_{1}} \geq 1,
$$

and so $f$ is a decreasing function. Set now $g(y)=\log f\left(e^{y}\right)$; by (3.6), we have

$$
-c_{1} u \leq g(y+u)-g(y) \leq-c_{2} u, \quad \forall u<0,
$$

or

$$
-c_{2} \leq \frac{g(y+u)-g(x)}{u} \leq-c_{1}, \quad \forall u<0
$$

Taking $u \rightarrow 0^{-}$, we obtain

$$
-c_{2} \leq g^{\prime}(y) \leq-c_{1}
$$

and hence

$$
c_{1} \leq \frac{-f^{\prime}\left(e^{y}\right)}{f\left(e^{y}\right)} e^{y} \leq c_{2} .
$$

Therefore,

$$
\frac{c_{1} f(x)}{x} \leq-f^{\prime}(x) \leq \frac{c_{2} f(x)}{x}
$$

and thus $f \in \mathfrak{V}$.

Corollary 3.4. If $f$ belongs to $\mathfrak{V}$, with constants $c_{1}$ and $c_{2}$, then

$$
f(t)=O\left(\frac{1}{t^{c_{2}}}\right), \quad t \longrightarrow 0^{+} .
$$

Proof. According to Theorem 3.3,

$$
t^{-c_{1}} \leq \frac{f(t)}{f(1)} \leq t^{-c_{2}}, \quad \forall t \in(0,1] .
$$

Thus,

$$
0<t^{c_{2}} f(t) \leq f(1), \quad \forall t \in(0,1]
$$

as required.

Note that the last corollary implies the integrability of $f(u) \sin (u x)$ (with respect to $u$ ), in any interval $(0, a), a<\infty$, only for $c_{2}<2$. Moreover, if $k$ is continuous on $(0, \infty)$ and

$$
k(t)=O\left(t^{\alpha}\right), \quad \text { as } t \longrightarrow 0,
$$


then for the integrability of $f(u) k(u x)$ at 0 it is sufficient to have $c_{2}<\alpha+1$. We observe also that the corollary implies that any $f \in \mathfrak{V}$ admits regularizations in the space $\mathscr{S}^{\prime}$ since $f(t)$ is bounded by a power of $t$ as $t \rightarrow 0^{+}$.

It is interesting that one may obtain inequalities similar to (3.6) and (3.7) for functions that do not belong to $\mathfrak{V}$. Indeed, the following result applies to oscillatory functions like $f(x)=x^{-c}(2+\sin \ln x)$.

THeOREM 3.5. Let $f$ be a positive function defined in $(0, \infty)$. Suppose that for each compact set $J \subset(0, \infty)$ there are constants $m=m(J)$ and $M=M(J)$ with $0<m<M$ such that

$$
m \leq \frac{f(u x)}{f(x)} \leq M, \quad \forall x \in(0, \infty), \forall u \in J .
$$

Then there exist constants $K_{q}, 1 \leq q \leq 4$, and $c_{1}, c_{2}$ such that

$$
\begin{aligned}
& \frac{K_{1}}{u^{c_{1}}} \leq \frac{f(u x)}{f(x)} \leq \frac{K_{2}}{u^{c_{2}}}, \quad \forall x \in(0, \infty), \forall u \in(0,1], \\
& \frac{K_{3}}{u^{c_{2}}} \leq \frac{f(u x)}{f(x)} \leq \frac{K_{4}}{u^{c_{1}}}, \quad \forall x \in(0, \infty), \forall u \in[1, \infty) .
\end{aligned}
$$

Proof. Let

$$
M_{+}(u)=\sup \left\{\frac{f(u x)}{f(x)}: x \in(0, \infty)\right\} .
$$

Then $M_{+}$is locally bounded in $(0, \infty)$ and satisfies

$$
M_{+}(u v) \leq M_{+}(u) M_{+}(v) .
$$

If we now write $\ln u=n+\theta$, where $n \in \mathbb{N}$ and where $0 \leq \theta<1$, for $u \geq 1$, we obtain

$$
M_{+}(u) \leq \sup \left\{M_{+}\left(e^{\theta}\right): 0 \leq \theta \leq 1\right\} M_{+}(e)^{\ln u},
$$

whenever $u \geq 1$, and thus the right inequality in (3.23) follows with $K_{2}=\sup \left\{M_{+}\left(e^{\theta}\right)\right.$ : $0 \leq \theta \leq 1\}$ and $c_{2}=-\ln \max \left\{M_{+}(e), 1\right\}$. This also gives us the left inequality in (3.24) with $K_{3}=1 / K_{2}$. The proof of the other two inequalities is similar (or can be obtained by applying what we already proved to the function $1 / f$ ).

\section{Oscillatory kernels}

Let $f \in \mathfrak{V}$. Suppose that $c_{2}<2$ in Definition 3.1. It was proved by Berndt [1] that its sine transform satisfies

$$
\frac{A}{x} f\left(\frac{1}{x}\right) \leq \int_{0}^{\infty} f(u) \sin (u x) d u \leq \frac{B}{x} f\left(\frac{1}{x}\right), \quad \forall x>0 .
$$

The previous inequality provides us an estimate of the global behavior for the sine transform of $f$ in terms of $f(1 / x)$. 
Our aim is to generalize (4.1) in two directions. First, we want to consider other kernels than sine, so we will give conditions on the kernel such that an estimate similar to (4.1) holds. Second, we will remove the condition $c_{2}<2$ for the sine transform; in such a case, the sine transform of $f$ will exist as a tempered distribution satisfying a global estimate as in (4.1), modulo a polynomial.

For our first goal, we define the $k$ transform of $f$ as the function $F$ given by

$$
F(x)=\int_{0}^{\infty} k(x u) f(u) d u .
$$

We will assume that $k$ satisfies

(1) $k$ is continuous on $[0, \infty)$;

(2) $k$ has only simple zeros, located at $t=\lambda_{n}$, where $\left\{\lambda_{n}\right\}_{n=0}^{\infty}$ satisfies that $\lambda_{0}=0$, and $\lambda_{0}<\lambda_{1}<\cdots<\lambda_{n}<\cdots$, where $\lambda_{n} \rightarrow \infty$ as $n \rightarrow \infty ; k$ changes sign at every $\lambda_{n}$, being positive on $\left(\lambda_{0}, \lambda_{1}\right)$, and

$$
\left|\int_{\lambda_{n}}^{\lambda_{n+1}} k(t) d t\right| \geq\left|\int_{\lambda_{n+1}}^{\lambda_{n+2}} k(t) d t\right| ;
$$

(3) $k(t)=O\left(t^{\alpha}\right), \alpha \geq 0, t \rightarrow 0$.

We can now state our first theorem.

Theorem 4.1. Let $f$ be an element of the class $\mathfrak{V}$. If $k$ satisfies (1), (2), and (3), and $c_{2}<$ $\alpha+1$, then

$$
F(x)=\frac{1}{x} f\left(\frac{1}{x}\right) h(x), \quad \forall x>0,
$$

where $h$ is continuous and bounded above and below by positive constants. Hence there exist positive constants $A$ and $B$ such that

$$
\frac{A}{x} f\left(\frac{1}{x}\right) \leq F(x) \leq \frac{B}{x} f\left(\frac{1}{x}\right), \quad \forall x>0
$$

Note that Theorem 4.1 is applicable to a wide class of kernels. For example, it applies to the Hankel kernel defined by

$$
k(t)=t^{1 / 2} J_{\nu}(t), \quad v>-\frac{1}{2},
$$

under the assumption $c_{2}<v+3 / 2$. Let us consider the proof of Theorem 4.1.

Proof. If we perform a change of variables we obtain

$$
F(x)=x^{-1} \int_{0}^{\infty} f\left(\frac{u}{x}\right) k(u) d u .
$$

Let

$$
d_{n}(x)=\int_{\lambda_{n}}^{\lambda_{n+1}} f\left(\frac{u}{x}\right) k(u) d u .
$$


8 Global behavior of integral transforms

It follows that

$$
F(x)=x^{-1} \sum_{n=0}^{\infty} d_{n}(x)
$$

Since $\sum_{n=0}^{\infty} d_{n}(x)$ is an alternating series and $\left|d_{n}(x)\right|$ decreases to zero as $n \rightarrow \infty$, we have

$$
x^{-1} \sum_{j=0}^{2 n+1} d_{j}(x) \leq F(x) \leq x^{-1} \sum_{j=0}^{2 n} d_{j}(x), \quad n \geq 0,
$$

which is equivalent to

$$
\int_{0}^{\lambda_{2 n+2}} \frac{f(u / x)}{f(1 / x)} k(u) d u \leq \frac{F(x)}{x^{-1} f(1 / x)} \leq \int_{0}^{\lambda_{2 n+1}} \frac{f(u / x)}{f(1 / x)} k(u) d u .
$$

In particular, for $n=0$,

$$
\int_{0}^{\lambda_{2}} \frac{f(u / x)}{f(1 / x)} k(u) d u \leq \frac{F(x)}{x^{-1} f(1 / x)} \leq \int_{0}^{\lambda_{1}} \frac{f(u / x)}{f(1 / x)} k(u) d u .
$$

Next, we will find positive constants $A, B<\infty$ such that

$$
\begin{aligned}
& \int_{0}^{\lambda_{1}} \frac{f(u / x)}{f(1 / x)} k(u) d u \leq B, \quad \forall x>0, \\
& \int_{0}^{\lambda_{2}} \frac{f(u / x)}{f(1 / x)} d u \geq A, \quad \forall x>0,
\end{aligned}
$$

and then (4.5) will follow. By Theorem 3.3,

$$
\frac{f(u / x)}{f(1 / x)} \leq \max \left\{\frac{1}{u^{c_{1}}}, \frac{1}{u^{c_{2}}}\right\}
$$

and hence

$$
\int_{0}^{\lambda_{1}} \frac{f(u / x)}{f(1 / x)} k(u) d u \leq \int_{0}^{\lambda_{1}} \max \left\{\frac{1}{u^{c_{1}}}, \frac{1}{u^{c_{2}}}\right\} k(u) d u
$$

If we set

$$
B=\int_{0}^{\lambda_{1}} \max \left\{\frac{1}{u^{c_{1}}}, \frac{1}{u^{c_{2}}}\right\} k(u) d u
$$


then (4.13) follows. Since $f$ is a decreasing function and $k$ is negative on $\left(\lambda_{1}, \lambda_{2}\right)$,

$$
\begin{aligned}
& \int_{0}^{\lambda_{1}} \frac{f(u / x)}{f(1 / x)} k(u) d u+\int_{\lambda_{1}}^{\lambda_{2}} \frac{f(u / x)}{f(1 / x)} k(u) d u \\
& \quad \geq \int_{0}^{\lambda_{1}} \frac{f(u / x)}{f(1 / x)} k(u) d u+\int_{\lambda_{1}}^{\lambda_{2}} \frac{f\left(\lambda_{1} / x\right)}{f(1 / x)} k(u) d u \\
& \quad=\int_{0}^{\lambda_{1}} \frac{\left(f(u / x)-f\left(\lambda_{1} / x\right)\right)}{f(1 / x)} k(u) d u+\frac{f\left(\lambda_{1} / x\right)}{f(1 / x)} \int_{0}^{\lambda_{2}} k(u) d u
\end{aligned}
$$

so that

$$
\int_{0}^{\lambda_{2}} \frac{f(u / x)}{f(1 / x)} k(u) d u \geq \int_{0}^{\lambda_{1}} \frac{\left(f(u / x)-f\left(\lambda_{1} / x\right)\right)}{f(1 / x)} k(u) d u .
$$

Therefore, applying the mean value theorem, we obtain

$$
f\left(\frac{u}{x}\right)-f\left(\frac{\lambda_{1}}{x}\right)=-f^{\prime}\left(\frac{\eta}{x}\right)\left(\frac{\lambda_{1}-u}{x}\right),
$$

for some point $\eta \in\left(u, \lambda_{1}\right)$. Then, by the left inequality in Definition 3.1,

$$
f\left(\frac{u}{x}\right)-f\left(\frac{\lambda_{1}}{x}\right) \geq c_{1} f\left(\frac{\eta}{x}\right) \frac{\lambda_{1}-u}{\eta} .
$$

Since $(1 / \eta) f(\eta / x) \geq\left(1 / \lambda_{1}\right) f\left(\lambda_{1} / x\right)$, we have

$$
f\left(\frac{u}{x}\right)-f\left(\frac{\lambda_{1}}{x}\right) \geq f\left(\frac{\lambda_{1}}{x}\right) \frac{c_{1}\left(\lambda_{1}-u\right)}{\lambda_{1}} \geq c_{1} f\left(\frac{\lambda_{1}}{x}\right) .
$$

Combining (4.19) and the last inequality, it follows that

$$
\int_{0}^{\lambda_{2}} \frac{f(u / x)}{f(1 / x)} k(u) d u \geq \frac{f\left(\lambda_{1} / x\right)}{f(1 / x)} \int_{0}^{\lambda_{1}} c_{1} k(u) d u .
$$

By Theorem 3.3, this implies that

$$
\int_{0}^{\lambda_{2}} \frac{f(u / x)}{f(1 / x)} k(u) d u \geq c_{1} \min \left\{\frac{1}{\lambda_{1}^{c_{1}}}, \frac{1}{\lambda_{1}^{c_{2}}}\right\} \int_{0}^{\lambda_{1}} k(u) d u .
$$

Setting $A$ equal to the right-hand side of the last inequality, the relation (4.14) has been proved.

Set now

$$
h(x)=\frac{F(x)}{x^{-1} f(1 / x)}, \quad x>0
$$

so that

$$
h(x)=\lim _{n \rightarrow \infty} \sum_{j=0}^{2 n} \frac{d_{j}(x)}{f(1 / x)}
$$


We will show that each $d_{j}$ is continuous. Pick $x_{0} \in(0, \infty)$ and choose $a$ such that $a>$ $\max \left\{x_{0}, 1\right\}$. By Theorem 3.3

$$
\left|f\left(\frac{u}{x}\right) k(u)\right| \leq \max \left\{x^{c_{1}}, x^{c_{2}}\right\} f(u) k(u),
$$

so that, for any $x \in(0, a]$, it follows that

$$
\left|f\left(\frac{u}{x}\right) k(u)\right| \leq a^{c_{2}} f(u)|k(u)|
$$

We have found an integrable function that dominates $f(u / x) k(u)$ for $x \in(0, a]$, this implies that

$$
\lim _{x \rightarrow x_{0}} d_{j}(x)=d_{j}\left(x_{0}\right)
$$

Finally, we show that $h$ is continuous. We claim that the convergence in (4.26) is uniform on each interval $[a, b], 0<a<b<\infty$. By $(4.10)$,

$$
\left|h(x)-\sum_{j=0}^{2 n} \frac{d_{j}(x)}{f(1 / x)}\right| \leq \frac{\left|d_{2 n+1}(x)\right|}{f(1 / x)} .
$$

We also have

$$
\begin{aligned}
\frac{\left|d_{2 n+1}(x)\right|}{f(1 / x)} & =\int_{\lambda_{2 n+1}}^{\lambda_{2 n+2}} \frac{f(u / x)}{f(1 / x)}|k(u)| d u \leq \frac{1}{f(1 / a)} \int_{\lambda_{2 n+1}}^{\lambda_{2 n+2}} f\left(\frac{u}{x}\right)|k(u)| d u \\
& \leq \frac{f\left(\lambda_{2 n+1} / b\right)}{f(1 / a)} \int_{\lambda_{2 n+1}}^{\lambda_{2 n+2}}|k(u)| d u \leq \frac{f\left(\lambda_{2 n+1} / b\right)}{f(1 / a)} \int_{0}^{\lambda_{1}} k(u) d u .
\end{aligned}
$$

Since the last term approaches to 0 as $n \rightarrow \infty$, the convergence in (4.26) is uniform on any interval $[a, b], 0<a<b<\infty$. Therefore, $h$ is continuous.

We now consider the second generalization of the estimate (4.1). We want to emphasize that the sine transform in this analysis will be considered as a tempered distribution, so that we will take a regularization of $f$, instead of $f$. If we let $c_{2}>2$ with no restriction, the sine transform of $f$ may not exist, as we remarked at the end of Section 3. In order to define a regularization of $f$, we need to extend $f$ to the whole real line; we do this by setting $f(x)=0$ for $x<0$; for the sake of simplicity, we will keep denoting this extension by $f$.

We state our second result.

Theorem 4.2. Let $f \in \mathfrak{V}$. Suppose that $\tilde{f}$ is any regularization of $f$ in $\mathscr{S}^{\prime}$ and denote the sine transform of $\tilde{f}$ by $F$. Then for all $x>0$ either

$$
F(x)=\frac{h(x)}{x} f\left(\frac{1}{x}\right)+P(x),
$$


or

$$
F(x)=-\frac{h(x)}{x} f\left(\frac{1}{x}\right)+P(x)
$$

where $h$ is continuous and bounded above and below by positive constants and $P$ is a polynomial.

Proof. It is known that any two regularizations of $f$, say $\tilde{f}$ and $\tilde{f}_{1}$, satisfy

$$
\tilde{f}(x)=\tilde{f}_{1}(x)+\sum_{i=0}^{m} a_{i} \delta^{(i)}(x)
$$

where $a_{0}, a_{1}, \ldots, a_{m}$ are some real constants. Observe that the sine transform of the sum of delta functions and its derivatives on the right-hand side is a polynomial. To see this fact, let $\phi$ be a test function of the space $\mathscr{Y}, k \in \mathbb{N}$; then,

$$
\begin{gathered}
\left\langle\delta^{(k)}(x), \int_{0}^{\infty} \phi(u) \sin (u x) d u\right\rangle=0, \quad \text { if } k \text { is even } ; \\
\left\langle\delta^{(k)}(x), \int_{0}^{\infty} \phi(u) \sin (u x) d u\right\rangle=\int_{0}^{\infty}(-x)^{k} \phi(x) d x, \quad \text { if } k=4 j+1 ; \\
\left\langle\delta^{(k)}(x), \int_{0}^{\infty} \phi(x) \sin (u x) d u\right\rangle=\int_{0}^{\infty} x^{k} \phi(x) d x, \quad \text { if } k=4 j+3 .
\end{gathered}
$$

Therefore, it suffices to work with any particular regularization of $f$. So we will find a regularization of $f$ for which the conclusion of the theorem holds. We will suppose that $c_{2} \geq 2$; otherwise, the conclusion of this theorem would be a consequence of Theorem 4.1. Let $n$ be the unique natural number such that

$$
2 n+1 \leq c_{2}<2 n+3
$$

We will divide the proof into two cases. We consider the cases when $n$ is odd and then when $n$ is even.

Assume first that $n$ is odd. Define now $\tilde{f}$ as

$$
\langle\tilde{f}(x), \phi(x)\rangle=\int_{0}^{2 \pi} f(x)\left(\phi(x)-\sum_{i=0}^{2 n+1} \frac{\phi^{(i)}(0)}{i !} x^{i}\right) d x+\int_{2 \pi}^{\infty} f(x) \phi(x) d x
$$

for $\phi \in \mathscr{Y}$. We will prove that $\tilde{f}$ is well defined. Let $\phi \in \mathscr{Y}$, then by Corollary 3.4,

$$
f(x)\left(\phi(x)-\sum_{i=0}^{2 n+1} \frac{\phi^{(i)}(0)}{i !} x^{i}\right)=O\left(x^{2 n+2-c_{2}}\right), \quad x \longrightarrow 0
$$

and so, by (4.36), it is integrable on $(0,2 \pi)$. The integrability on $(2 \pi, \infty)$ is clear since $\phi \in \mathscr{Y}$. By a standard argument, $\tilde{f} \in \mathscr{S}^{\prime}$. 
We will prove the formula for the sine transform of $\tilde{f}$. Denote by $\tilde{F}$ the sine transform of $\tilde{f}$. Let us now set

$$
K(x)=\sin x-\sum_{i=0}^{n} \frac{(-1)^{i}}{(2 i+1) !} x^{2 i+1} .
$$

Since $n$ is odd,

$$
K(x) \geq 0, \quad \text { for } x \geq 0 .
$$

Using the definition of $\tilde{F}$, we have for $x>0$,

$$
\begin{aligned}
\langle\tilde{F}(x), \phi(x)\rangle= & \left\langle\tilde{f}(x), \int_{0}^{\infty} \phi(u) \sin (x u) d u\right\rangle=\int_{0}^{2 \pi} f(x)\left(\int_{0}^{\infty} \phi(u) K(x u) d u\right) d x \\
& +\int_{2 \pi}^{\infty} f(x)\left(\int_{0}^{\infty} \phi(u) \sin (x u) d u\right) d x \\
= & \int_{0}^{\infty} \frac{\phi(x)}{x}\left(\int_{0}^{2 \pi} f\left(\frac{u}{x}\right) K(u) d u+\int_{2 \pi}^{\infty} f\left(\frac{u}{x}\right) \sin u d u\right) d x,
\end{aligned}
$$

for every $\phi \in \mathscr{Y}$. It follows that if $x>0$,

$$
\widetilde{F}(x)=\frac{1}{x}\left[\int_{0}^{2 \pi} f\left(\frac{u}{x}\right) K(u) d u+\int_{2 \pi}^{\infty} f\left(\frac{u}{x}\right) \sin u d u\right] .
$$

Hence $\widetilde{F}$ can be identified with a classical function, in the sense that $\widetilde{F}$ is the distribution generated by the function given by (4.42).

Next we set

$$
h(x)=\frac{\tilde{F}(x)}{x^{-1} f(1 / x)}, \quad \text { for } x>0 .
$$

We will find two constants, $A$ and $B$, so that

$$
A \leq h(x) \leq B, \quad x>0
$$

Notice that

$$
h(x)-\int_{0}^{2 \pi} \frac{f(u / x)}{f(1 / x)} K(u) d u=\int_{2 \pi}^{\infty} \frac{f(u / x)}{f(1 / x)} \sin u d u .
$$

We also have that

$$
\int_{2 \pi}^{4 \pi} \frac{f(u / x)}{f(1 / x)} \sin u d u \leq \int_{2 \pi}^{\infty} \frac{f(u / x)}{f(1 / x)} \sin u d u \leq \int_{2 \pi}^{3 \pi} \frac{f(u / x)}{f(1 / x)} \sin u d u .
$$

We can apply the argument that we used in Theorem 4.1 to find positive constants $A^{\prime}$ and $B^{\prime}$ such that

$$
\int_{2 \pi}^{3 \pi} \frac{f(u / x)}{f(1 / x)} \sin u d u \leq B^{\prime}, \quad A^{\prime} \leq \int_{2 \pi}^{4 \pi} \frac{f(u / x)}{f(1 / x)} \sin u d u
$$


for all $x \in(0, \infty)$. Using the last inequalities, we obtain that

$$
A^{\prime} \leq \int_{2 \pi}^{\infty} \frac{f(u / x)}{f(u / x)} \sin u d u \leq B^{\prime}
$$

It follows that

$$
\int_{0}^{2 \pi} \min \left\{\frac{1}{u^{c_{1}}}, \frac{1}{u^{c_{2}}}\right\} K(u) d u+A^{\prime} \leq h(x), \quad h(x) \leq \int_{0}^{2 \pi} \max \left\{\frac{1}{u^{c_{1}}}, \frac{1}{u^{c_{2}}}\right\} K(u) d u+B^{\prime},
$$

which shows that $h$ is bounded above and below by positive constants.

We now prove the continuity of $h$. The continuity of

$$
\int_{2 \pi}^{\infty} f\left(\frac{u}{x}\right) \sin u d u
$$

follows from the proof of Theorem 4.1. Moreover, since

$$
\frac{f(u / x)}{f(1 / x)} K(u) \leq \max \left\{\frac{1}{u^{c_{1}}}, \frac{1}{u^{c_{2}}}\right\} K(u),
$$

it follows by the Lebesgue dominated convergence theorem that

$$
h(x)-\int_{2 \pi}^{\infty} \frac{f(u / x)}{f(1 / x)} \sin u d u
$$

is continuous, and so is $h(x)$. This completes the proof for the odd case.

We now assume that $n$ is an even number. Define $\tilde{f}$ as

$$
\langle\tilde{f}(x), \phi(x)\rangle=\int_{0}^{3 \pi} f(x)\left(\phi(x)-\sum_{i=0}^{2 n+1} \frac{\phi^{(i)}(0)}{i !} x^{i}\right) d x+\int_{3 \pi}^{\infty} f(x) \phi(x) d x
$$

for $\phi \in \mathscr{S}$. It follows that $\tilde{f} \in \mathscr{S}^{\prime}$. Set

$$
J(x)=\sum_{i=0}^{n} \frac{(-1)^{i}}{(2 i+1) !} x^{2 i+1}-\sin x
$$

which is a positive function, since $n$ is an even number. Let $\tilde{F}$ be the sine transform of $\tilde{f}$. We have that if $x>0$,

$$
\widetilde{F}(x)=\frac{1}{x}\left[-\int_{0}^{3 \pi} f\left(\frac{u}{x}\right) J(u) d u+\int_{3 \pi}^{\infty} f\left(\frac{u}{x}\right) \sin u d u\right] .
$$

Set

$$
h(x)=-\frac{\tilde{F}(x)}{x^{-1} f(1 / x)}, \quad x>0 .
$$


It follows that

$$
h(x)=\int_{0}^{3 \pi} \frac{f(u / x)}{f(1 / x)} J(u) d u-\int_{3 \pi}^{\infty} \frac{f(u / x)}{f(1 / x)} \sin u d u
$$

for $x>0$. We can find two positive constants, $A^{\prime \prime}$ and $B^{\prime \prime}$, such that

$$
-\int_{3 \pi}^{4 \pi} \frac{f(u / x)}{f(1 / x)} \sin u d u \leq B^{\prime \prime}, \quad-\int_{3 \pi}^{5 \pi} \frac{f(u / x)}{f(1 / x)} \sin u d u \geq A^{\prime \prime} .
$$

From these inequalities, it follows that

$$
\int_{0}^{3 \pi} \min \left\{\frac{1}{u^{c_{1}}}, \frac{1}{u^{c_{2}}}\right\} J(u) d u+A^{\prime \prime} \leq h(x), \quad h(x) \leq \int_{0}^{3 \pi} \max \left\{\frac{1}{u^{c_{1}}}, \frac{1}{u^{c_{2}}}\right\} J(u) d u+B^{\prime \prime},
$$

which proves the required inequalities. The continuity of $h$ can be established as in the odd case.

\section{Laplace transform}

In this section, we will give a result analogous to Theorem 4.2 for the Laplace transform. The estimate is as follows.

Theorem 5.1. Let $f \in \mathfrak{V}$. Suppose that $\tilde{f}$ is any regularization of $f$ in $\mathscr{S}^{\prime}$ and denote its Laplace transform by $L$. Then for all $x>0$, either

$$
L(x)=\frac{h(x)}{x} f\left(\frac{1}{x}\right)+P(x)
$$

or

$$
L(x)=-\frac{h(x)}{x} f \frac{1}{x}+P(x)
$$

where $h$ is continuous and bounded above and below by positive constants, and $P$ is a polynomial.

Proof. We proceed as in Theorem 4.2. It suffices to consider a particular regularization of $f$. Let $n$ be the integer part of $c_{2}$. We will consider two cases. First, we assume that $n$ is odd, and then we consider the even case.

Assume that $n$ is odd. Define $\tilde{f}$ as

$$
\langle\tilde{f}(x), \phi(x)\rangle=\int_{0}^{1} f(x)\left(\phi(x)-\sum_{i=0}^{n} \frac{\phi^{i}(0)}{i !}\right) d x+\int_{1}^{\infty} f(x) \phi(x) d x,
$$


for $\phi \in \mathscr{Y}$. Then, $\tilde{f}$ is a regularization of $f$ in $\mathscr{Y}^{\prime}$. Since supp $\tilde{f}=[0, \infty)$, its Laplace transform is well defined. Let us denote its Laplace transform by $\widetilde{L}$, so that

$$
\begin{aligned}
\widetilde{L}(x) & =\int_{0}^{1} f(u)\left(e^{-u x}-\sum_{i=0}^{n} \frac{(-u x)^{i}}{i !}\right) d u+\int_{1}^{\infty} f(u) e^{-u x} d u \\
& =\frac{1}{x}\left[\int_{0}^{1} f\left(\frac{u}{x}\right)\left(e^{-u}-\sum_{i=0}^{n} \frac{(-u)^{i}}{i !}\right) d u+\int_{1}^{\infty} f\left(\frac{u}{x}\right) e^{-u} d u\right] .
\end{aligned}
$$

We now consider the following inequality:

$$
e^{-x}-\sum_{i=0}^{n} \frac{(-x)^{i}}{i !}>0, \quad \text { for } x>0 \text {. }
$$

Set

$$
h(x)=\frac{\tilde{L}(x)}{x^{-1} f(1 / x)}, \quad K(x)=e^{-x}-\sum_{i=0}^{n} \frac{(-x)^{i}}{i !} .
$$

Then, we have

$$
\int_{0}^{1} \frac{K(u)}{u^{c_{1}}} d u+\int_{1}^{\infty} \frac{e^{-u}}{u^{c_{2}}} d u \leq h(x) \leq \int_{0}^{1} \frac{K(u)}{u^{c_{2}}} d u+\int_{0}^{\infty} \frac{e^{-u}}{u^{c_{1}}} d u .
$$

This completes the proof for the odd case.

Assume now that $n$ is even. Set

$$
J(x)=\sum_{i=0}^{n} \frac{(-x)^{i}}{i !}-e^{-x}
$$

it follows that

$$
J(x)>0, \quad \text { for } x>0 .
$$

Take $A>1$ such that

$$
\int_{0}^{1} \frac{J(u)}{u^{c_{1}}} d u-\int_{A}^{\infty} \frac{e^{-u}}{u^{c_{1}}} d u>0, \quad \int_{0}^{1} \frac{J(u)}{u^{c_{2}}} d u-\int_{A}^{\infty} \frac{e^{-u}}{u^{c_{2}}} d u>0 .
$$

We define $\tilde{f}$, a regularization of $f$, as

$$
\langle\tilde{f}(x), \phi(x)\rangle=\int_{0}^{A} f(x)\left(\phi(x)-\sum_{i=0}^{n} \frac{\phi^{(i)}(0)}{i !}\right) d x+\int_{A}^{\infty} f(x) \phi(x) d x .
$$

It follows that $\tilde{L}$, the Laplace transform of $\tilde{f}$, is given by

$$
\tilde{L}(x)=\frac{1}{x}\left(-\int_{0}^{A} f\left(\frac{u}{x}\right) J(u) d u+\int_{A}^{\infty} f\left(\frac{u}{x}\right) e^{-u} d u\right) .
$$


Define now $h$ by

$$
h(x)=\frac{-\tilde{L}(x)}{x^{-1} f(1 / x)}
$$

We have that

$$
\begin{aligned}
& \int_{0}^{1} \frac{J(u)}{u^{c_{1}}} d u+\int_{1}^{A} \frac{J(u)}{u^{c_{2}}} d u-\int_{A}^{\infty} \frac{e^{-u}}{u^{c_{1}}} d u \leq h(x), \\
& h(x) \leq \int_{0}^{1} \frac{J(u)}{u^{c_{2}}} d u+\int_{1}^{A} \frac{J(u)}{u^{c_{1}}} d u-\int_{A}^{\infty} \frac{e^{-u}}{u^{c_{2}}} d u,
\end{aligned}
$$

so $h$ is bounded above and below by positive constants.

\section{References}

[1] R. Berndt, A formula for the Fourier transform of certain odd differentiable functions, Journal of Mathematical Analysis and Applications 285 (2003), no. 2, 349-355.

[2] _ Singular integrals with new singularities, Dissertation, University of Minnesota, Minnesota, 2003.

[3] R. Estrada, Regularization of distributions, International Journal of Mathematics and Mathematical Sciences 21 (1998), no. 4, 625-636.

[4] R. Estrada and R. P. Kanwal, A Distributional Approach to Asymptotics. Theory and Applications, 2nd ed., Birkhäuser Advanced Texts: Basel Textbooks, Birkhäuser Boston, Massachusetts, 2002.

[5] R. P. Kanwal, Generalized Functions. Theory and Technique, 2nd ed., Birkhäuser Boston, Massachusetts, 1998.

[6] E. Seneta, Regularly Varying Functions, Lecture Notes in Mathematics, vol. 508, Springer, Berlin, 1976.

[7] K. Soni and R. P. Soni, Slowly varying functions and asymptotic behavior of a class of integral transforms. I, Journal of Mathematical Analysis and Applications 49 (1975), no. 1, 166-179.

[8] _L Slowly varying functions and asymptotic behavior of a class of integral transforms. II, Journal of Mathematical Analysis and Applications 49 (1975), no. 1, 477-495.

[9] __ Slowly varying functions and asymptotic behavior of a class of integral transforms. III, Journal of Mathematical Analysis and Applications 49 (1975), no. 3, 612-628.

[10] F. Trèves, Topological Vector Spaces, Distributions and Kernels, Academic Press, New York, 1967.

[11] A. H. Zemanian, Distribution Theory and Transform Analysis. An Introduction to Generalized Functions, with Applications, 2nd ed., Dover, New York, 1987.

Jasson Vindas: Mathematics Department, Louisiana State University, Baton Rouge, LA 70803, USA E-mail address: jvindas@math.lsu.edu

Ricardo Estrada: Mathematics Department, Louisiana State University, Baton Rouge, LA 70803, USA E-mail address: restrada@math.lsu.edu 